\title{
Pazopanib for the Treatment of Non-clear Cell Renal Cell Carcinoma: A Single-Arm, Open-Label, Multicenter, Phase II Study
}

\section{Ki Sun Jung, MD 1 \\ Su Jin Lee, MD, $P h D^{1}$ \\ Se Hoon Park, MD, PhD' \\ Jae-Lyun Lee, MD, PhD² \\ Se-Hoon Lee, MD, $\mathrm{PhD}^{3}$ \\ Jae Yun Lim, MD, PhD ${ }^{4}$ \\ Jung Hun Kang, MD, $P h D^{5}$ \\ Suee Lee, MD, $P h D^{6}$ \\ Sun Young Rha, $\mathrm{MD}, \mathrm{PhD}^{7}$ \\ Kyung Hee Lee, MD, $\mathrm{PhD}{ }^{8}$ \\ Ho Young Kim, MD, PhD ${ }^{9}$ \\ Ho Yeong Lim, MD, PhD'}

*A list author's affiliations appears at the end of the paper.
Correspondence: Ho Yeong Lim, MD, PhD Division of Hematology and Oncology, Department of Medicine, Samsung Medical Center, Sungkyunkwan University School of Medicine, 81 Irwon-ro, Gangnam-gu, Seoul 06351, Korea

Tel: 82-2-3410-0914

Fax: 82-2-3410-1754

E-mail: hoylim@skku.edu

Received December 8, 2016

Accepted May 16, 2017

Published Online May 22, 2017

\section{Purpose}

The optimal treatment strategy for patients with metastatic non-clear cell type renal cell carcinoma (nccRCC) remains unclear. Although several inhibitors of vascular endothelial growth factor have recently shown efficacy against nccRCC, the clinical benefit of pazopanib in nccRCC has not been analyzed. We therefore designed a single-arm, open-label, phase II study to determine the efficacy and safety of pazopanib in patients with nccRCC.

\section{Materials and Methods}

Patients with locally advanced or metastatic nccRCC, except for collecting duct or sarcomatoid type, received $800 \mathrm{mg} /$ day of pazopanib daily until progression of disease or intolerable toxicity. One cycle was defined as 4 weeks and tumor response was evaluated every two cycles. The primary objective was overall response rate (ORR).

\section{Results}

A total of 29 eligible patients were enrolled at nine centers in Korea from December 2012 and September 2014. The median age of the patients was 58 years (range, 27 to 76 years) and 21 patients (72\%) were male. Regarding histology type, 19 patients had papillary, three had chromophobe, two had unclassified and five had unknown non-clear cell type. Of 28 evaluable patients, eight achieved a confirmed partial response with ORR of $28 \%$. The median progression-free survival was 16.5 months (95\% confidence interval, 10.9 to 22.1 ) and median overall survival was not reached. Sixteen patients (55\%) experienced treatment-related toxicity of grade 3 or more, but most adverse events were overcome through dose reduction and delay.

\section{Conclusion}

In this prospective phase II study, pazopanib demonstrated promising activity and tolerable safety profile in patients with metastatic nccRCC.

\section{Key words}

Non-clear cell renal cell carcinoma, Pazopanib, Phase II study 


\section{Introduction}

Non-clear cell type renal cell carcinoma (nccRCC) is a heterogeneous mixture of diverse histologies including papillary, chromophobe, collecting duct, medullary and unclassified renal cell carcinoma (RCC). It usually accounts for approximately $20 \%$ of all RCC cases [1] and shows poor treatment response to systemic therapy and poor survival in comparison with clear cell RCC (ccRCC) $[2,3]$.

Systemic therapy is the main treatment option for metastatic RCC and various kinds of therapy with strong evidence, such as sunitinib, pazopanib, and axitinib, are available for ccRCC [4]. However, there is little evidence and fewer available options for nccRCC. Temsirolimus is currently the preferred treatment for metastatic nccRCC and is especially recommended as an initial targeted agent for patients classified as poor risk according to the Memorial Sloan-Kettering Cancer Center (MSKCC) risk group [5,6]. However, the evidence for this recommendation originated in a previous phase III trial of temsirolimus versus interferon $\alpha$ that included patients with poor-risk metastatic RCC of all histologic type and showed a clinical benefit of temsirolimus. For patients in the other risk groups, there is a lack of evidence for the use of targeted agents.

Pazopanib is a multi-targeted receptor tyrosine kinase inhibitor (TKI) that inhibits tumor cell proliferation and angiogenesis through selective inhibition of vascular endothelial growth factor receptors (VEGFRs), plateletderived growth factor receptors, and c-kit $[7,8]$. Several clinical trials [9-11] have analyzed the efficacy and safety for this pazopanib and it is currently accepted as a first-line therapeutic option in patients with metastatic ccRCC. In patients with ccRCC, sunitinib and pazopanib showed similar efficacy, but pazopanib had lower toxicity than sunitinib [10]. In addition, pazopanib showed treatment response in patients with ovarian cancer, soft tissue sarcoma and nonsquamous cell lung cancer [12-14].

Recently, sunitinib and sorafenib showed efficacy for patients with metastatic nccRCC in some reports, including an expanded-access trial $[15,16]$. Specially, sunitinib showed a high overall response rate (ORR) of 36\% [17]. Although there is one retrospective study [18], the efficacy of pazopanib has not been prospectively surveyed in patients with nccRCC. Therefore, we designed a single-arm, open-label, phase II study to evaluate the efficacy and safety of pazopanib in patients with nccRCC.

\section{Materials and Methods}

\section{Study design}

This was an open-label, phase II, multicenter study conducted at nine centers in Korea. The protocol was approved by the institutional review board of each participating center, and the trial was conducted in accordance with the principles of the Declaration of Helsinki. All patients provided written informed consent before enrollment. GlaxoSmithKline (GSK; Seoul, Korea) provided pazopanib but was not involved in patient accrual, data analysis, or manuscript preparation. The trial was registered at ClinicalTrials.gov (NCT01538238).

\section{Patient selection}

Inclusion criteria were as follows: histologically confirmed RCC with more than 50\% non-clear cell histologic component, including papillary type, chromophobe type, or unclassified cell types (excluding collecting duct and sarcomatoid type, but including tumors with sarcomatoid type or collecting duct type cells if they were less than $5 \%$ of the total cancer tumor); at least one measurable lesion by Response Evaluation Criteria in Solid Tumors (RECIST) ver. 1.1 that had not been previously treated with radiotherapy; Eastern Cooperative Oncology Group performance status of 0 or 1 ; and adequate hepatic, renal and hematologic function. Patients who previously received angiogenesis inhibitors (such as sunitinib, sorafenib, or bevacizumab), mesenchymal-epithelial transition factor inhibitors or c-kit inhibitors for the treatment of RCC were excluded. However, patients who completed immune therapy (interleukin 2, interferon) at least 6 weeks previously or treatment with mammalian target of rapamycin (mTOR) inhibitor (everolimus, temsirolimus) at least 3 weeks previously could be enrolled in this study.

\section{Treatment}

Patients received $800 \mathrm{mg}$ oral pazopanib once daily in 4-week treatment cycles until disease progression or unacceptable toxicity. Toxicities were evaluated according to the National Cancer Institute's Common Toxicity Criteria ver. 3.0. Grade 2 nonhematologic and grade 3 hematologic toxicities were managed by cessation of the drug until resolution to grade 1 and then resuming treatment without a dose reduction. If the patient experienced a second toxicity, the drug dosage was reduced to $600 \mathrm{mg}$ once daily. Grade 3 or 4 hepatic toxicities were managed through dose interruption followed by dose reduction to $600 \mathrm{mg}$ once daily. If dose reduction of more than $400 \mathrm{mg}$ was required, patients were taken off the study. 


\section{Objectives and response evaluation}

The primary endpoint was ORR, and secondary endpoints were progression-free survival (PFS), overall survival (OS), and safety. Radiographic imaging and tumor assessments were performed every 8 weeks based on RECIST 1.1 criteria [4]. All complete responses (CRs) and partial responses (PRs) were confirmed by repeated computed tomography (CT) scan evaluation per RECIST ver. 1.1. MSKCC risk group was defined based on 2002 risk model criteria [19].

\section{Statistical considerations}

A Simon's two-stage phase II optimal design was used to assess the primary endpoint of response according to the following parameters: reference response rate of $10 \%$; experimental response rate of 30\%; and type I and II error rates of $5 \%$ and $20 \%$, respectively. Ten patients were treated at the first stage and the study would be stopped early if fewer than two patients responded; otherwise, the study would proceed to the second stage, in which an additional 19 patients would be treated. If six or more responders were observed from the cumulative 29 patients, pazopanib would be considered worthy of further testing in this study population.

Standard descriptive and analytical methods were used to describe the patient population and their baseline characteristics and clinical outcomes. PFS and OS were estimated using the Kaplan-Meier method. We also used the Cox proportional hazards regression models to identify predictive factors of PFS and OS. Statistical data were analyzed using SPSS ver. 18 (IBM Corp., Armonk, NY, http://www.ibm. com).

\section{Results}

\section{Patient characteristics}

A total of 29 patients were enrolled between December 2012 and September 2014. The baseline patient characteristics are presented in Table 1 . The median age was 58 years (range, 27 to 76 years) and most patients were male $(72 \%)$. Nineteen patients $(66 \%)$ had papillary type and three patients $(10 \%)$ had chromophobe type. Twenty-one patients $(72 \%)$ were classified as MSKCC favorable risk, six $(21 \%)$ as intermediate risk and two $(7 \%)$ as poor risk. Twenty patients $(69 \%)$ underwent prior nephrectomy: 14 (70\%) for curative purpose and six (30\%) for cytoreductive purpose. Two patients $(7 \%)$ received immunotherapy and 12 patients $(41 \%)$ were treated with mTOR inhibitors prior to this study. The median duration of
Table 1. Baseline patient characteristics

\begin{tabular}{|c|c|}
\hline Characteristic & No. of patients $(\%)(\mathrm{n}=29)$ \\
\hline Age, median (range, yr) & $58(27-76)$ \\
\hline \multicolumn{2}{|l|}{ Sex } \\
\hline Male & $21(72)$ \\
\hline Female & $8(28)$ \\
\hline \multicolumn{2}{|l|}{ Smoking history } \\
\hline Never smoker & $15(52)$ \\
\hline Ex-smoker & $10(34)$ \\
\hline Current smoker & $4(14)$ \\
\hline \multicolumn{2}{|c|}{ ECOG performance status } \\
\hline 0 & $13(45)$ \\
\hline 1 & $16(55)$ \\
\hline \multicolumn{2}{|l|}{ Histology } \\
\hline Papillary & $19(66)$ \\
\hline Chromophobe & $3(10)$ \\
\hline Unclassified & $2(7)$ \\
\hline Unknown & $5(17)$ \\
\hline \multicolumn{2}{|l|}{ MSKCC risk group } \\
\hline Favorable & $21(72)$ \\
\hline Intermediate & $6(21)$ \\
\hline Poor & $2(7)$ \\
\hline \multicolumn{2}{|c|}{ Most common site of metastasis } \\
\hline Lung & $8(28)$ \\
\hline Lymph node & $13(45)$ \\
\hline Bone & $14(48)$ \\
\hline Liver & $6(21)$ \\
\hline \multicolumn{2}{|l|}{ No. of organs involved } \\
\hline 1 & $13(45)$ \\
\hline 2 & $4(14)$ \\
\hline$\geq 3$ & $12(41)$ \\
\hline Prior nephrectomy & $20(69)$ \\
\hline Curative & $14(70)$ \\
\hline Cytoreductive & $6(30)$ \\
\hline \multicolumn{2}{|l|}{ Prior treatment } \\
\hline RT & $8(28)$ \\
\hline Immunotherapy & $2(7)$ \\
\hline mTOR inhibitor & $12(41)$ \\
\hline None & $13(45)$ \\
\hline
\end{tabular}

ECOG, Eastern Cooperative Oncology Group; MSKCC, Memorial Sloan-Kettering Cancer Center; RT, radiotherapy; mTOR, mammalian target of rapamycin.

pazopanib treatment was 8.8 months (range, 0.8 to 27.8 months).

\section{Response and survival}

One patient discontinued pazopanib before CT response evaluation because of death as a result of disease deterioration. No patient showed CR, eight patients showed PR and 17 
Table 2. Summary of tumor response

\begin{tabular}{lc} 
Best response & No. of patients $(\%)(\mathbf{n}=\mathbf{2 8})$ \\
CR & 0 \\
PR & $8(28)$ \\
SD & $17(61)$ \\
PD & $3(11)$ \\
Response rate (CR+PR) & $8(28)$ \\
Disease control rate (CR+PR+SD) & $25(89)$ \\
\hline
\end{tabular}

$\mathrm{CR}$, complete response; $\mathrm{PR}$, partial response; $\mathrm{SD}$, stable disease; $\mathrm{PD}$, progressive disease.

patients showed stable disease (SD), resulting in an ORR of $28 \%$ (95\% confidence interval [CI], 12 to 44 ) and disease control rate (DCR) of $89 \%$ (95\% CI, 78 to 100) (Table 2). After a median follow-up period of 21.3 months, the median PFS was 16.5 months (95\% CI, 10.9 to 22.1). Median OS has not been reached yet with a 1-year survival rate of $69 \%$ (Fig. 1). According to histologic type, ORR was 39\% for papillary type, 33\% for chromophobe type, and patients with unclassified type RCC did not show any response (Table 3). During the followup period, disease progression were observed in 17 patients $(59 \%)$ and 12 patients $(41 \%)$ died. Causes of death were disease progression (10 patients), pneumonia (one patient), and microperforation of small bowel (one patient). In survival analysis for PFS and OS, only MSKCC risk group $(\mathrm{p}<0.001)$ was a predictive factor for OS (S1 Table).

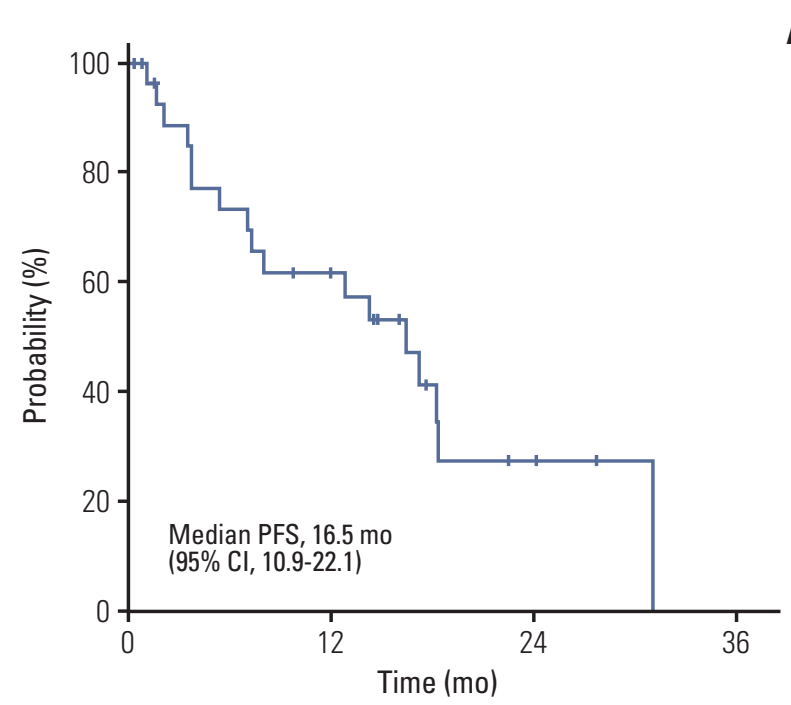

\section{Adverse events}

Twenty-eight patients (97\%) experienced one or more adverse events (AEs) (Table 4). The most common AEs were hypertension (66\%), nausea/ vomiting (59\%), hair color change $(59 \%)$, diarrhea $(55 \%)$, anorexia $(48 \%)$, mucositis $(45 \%)$, and abdominal pain $(41 \%)$. The most common laboratory abnormalities were hepatic dysfunction (45\%) and hematologic dysfunction (anemia 17\%, leukopenia 14\%, thrombocytopenia $24 \%)$. Sixteen patients $(55 \%)$ had AEs of grade $\geq 3$. The most common grade $\geq 3 \mathrm{AEs}$ were hepatic dysfunction (24\%), thrombocytopenia $(10 \%)$, and abdominal pain $(10 \%)$. Dose reduction and delays due to AEs occurred in $19(66 \%)$ and 17 (59\%) patients, respectively. Most AEs were overcome through dose reduction and/or delay; only one patient stopped treatment with pazopanib because of grade $\geq 3$ hepatic dysfunction. Treatment-related mortality was observed in one patient ( $3 \%$, pneumonia).

\section{Discussion}

In patients with clear cell type histology of metastatic RCC, many studies have been performed on treatment for first-line therapy as well as subsequent therapy and several therapeutic agents are available as treatment options in these patients. However, the optimal therapy for nccRCC type remains unclear. Thus, enrollment of patients with nccRCC type to clinical trial is required according to National Comprehen-
A

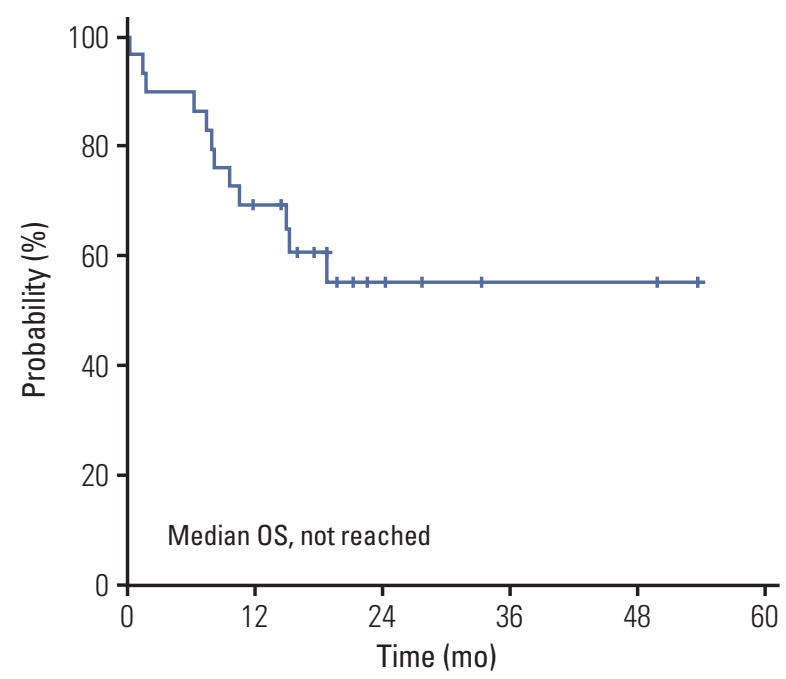

Fig. 1. Progression-free survival (PFS) (A) and overall survival (OS) (B) of 28 patients. CI, confidence interval. 
Table 3. Response and survival according to histologic type $(n=28)$

\begin{tabular}{lcccc} 
Histologic type & Papillary $(\mathrm{n}=\mathbf{1 8})$ & Chromophobe $(\mathrm{n}=\mathbf{3})$ & Unclassified $(\mathrm{n}=\mathbf{2})$ & Unknown $(\mathrm{n}=\mathbf{5})$ \\
Response & & & & \\
PR & 7 & 1 & - & - \\
SD & 9 & 2 & 2 & 4 \\
PD & 2 & - & - & 1 \\
ORR (\%) & 39 & 18.3 & 0 & 0 \\
PFS & 17.3 & $(11.9-24.7)$ & - & 5.4 \\
(95\% CI, mo) & $(14.8-19.8)$ & 18.9 & 7.6 & $(1.8-9.0)$ \\
OS & Not reached & - & - & 6.4 \\
$\mathbf{9 5 \%}$ CI, mo) & - & $0-16.3)$ \\
\hline
\end{tabular}

$\mathrm{PR}$, partial response; $\mathrm{SD}$, stable disease; $\mathrm{PD}$, progressive disease; $\mathrm{ORR}$, overall response rate; $\mathrm{PFS}$, progression-free survival; $\mathrm{CI}$, confidence interval; OS, overall survival.

sive Cancer Network guidelines [4]. As a result, treatment strategy of metastatic nccRCC is currently challenging and various studies should be conducted to demonstrate clinical efficacy of available therapeutic options in these patients.

Temsirolimus is a first-line therapeutic agent that could be considered for patients with metastatic nccRCC. In subgroup analysis of the global advanced RCC trial, the median OS was 11.6 months with temsirolimus and 4.3 months with interferon-alpha for nccRCC patients $[5,6]$. However, this trial enrolled patients with clear cell type as well as non-clear cell type and only included poor-risk patients according to MSKCC risk criteria. In phase II trial of everolimus, another mTOR inhibitor, the ORR was $10.2 \%$ and median PFS was 5.2 months in patients with metastatic nccRCC who previously received a VEGFR-TKI [20].

In the case of TKIs, various therapeutic agents have showed clinical efficacy for ccRCC patients and are currently used as standard therapy. For this reason, several TKIs have been tested in metastatic nccRCC and there are reports of the use of sunitinib, sorafenib, and erlotinib in these patients. All of these studies were phase II or expanded-access trials, and the phase III trial of TKIs in this setting has not been surveyed yet. In the phase II trial $(\mathrm{n}=31)$ for sunitinib, median PFS was 6.4 months and ORR was 36\% [17]. In another study of treatment with sunitinib or sorafenib, ORR was $23 \%$ and median PFS was 10.6 months ( $n=53)$ [16]. A phase II study of erlotinib in patients with papillary RCC $(\mathrm{n}=45)$ showed an ORR of $11 \%$, DCR of $64 \%$, and median OS of 27 months [21]. Several clinical trials using pazopanib or axitinib in patients with metastatic nccRCC are currently ongoing.

In this study, we analyzed the clinical efficacy and safety of pazopanib in patients with metastatic nccRCC. As a result, the ORR was $28 \%$ and DCR was $89 \%$. The median PFS was 16.5 months (95\% CI, 10.9 to 22.1) and 1-year OS rate of $69 \%$. This is the first report of the use of pazopanib in patients with metastatic nccRCC and showed promising efficacy in these patients. Based on these findings, further research is required to define the optimal treatment for patients with advanced nccRCC.

This meaningful efficacy seems to result from the fact that all patients had good performance status and many were classified as MSKCC favorable (72\%) or intermediate (21\%) risk groups. In a previous study, temsirolimus showed a survival benefit in the poor risk group [6]. Also, in another study, the median PFS was longer with sunitinib than with everolimus in good or intermediate risk group of nccRCC patients, but median PFS was longer with everolimus than with sunitinib for the poor risk group [22]. The enrolled patients of our study included a relatively high proportion in favorable or intermediate risk groups compared with other studies, and this might increase the efficacy of pazopanib. With regard to subtypes of nccRCC, papillary type 1 and chromophobe RCC tend to have an indolent disease course, whereas collecting duct and unclassified subtypes are associated with poor outcomes [23,24]. Although only a small number of patients were included in this study, papillary and chromophobe types showed a treatment response to pazopanib, whereas the unclassified type group had no responders. Further studies of the optimal treatment according to each risk group and histologic subtype in patients with nccRCC are needed.

Most patients (97\%) suffered AEs related to pazopanib treatment during the follow-up period and AEs of grade $\geq 3$ were reported in $55 \%$ of patients in this study. However, these AEs were almost all clinically manageable through dose modification or interruption, with the exception of only one patient. Treatment-related mortality was confirmed in $3 \%$ of our patients (one patient, who died from pneumonia). Although one patient died due to microperforation of small bowel, which was a serious and unexpected $\mathrm{AE}$, we consid- 
Table 4. Treatment-related adverse events $(n=29)$

\begin{tabular}{|c|c|c|}
\hline Adverse event & All grades & Grade $\geq 3$ \\
\hline \multicolumn{3}{|l|}{ Symptoms and signs } \\
\hline Anorexia & $14(48)$ & $2(7)$ \\
\hline Mucositis & $13(45)$ & - \\
\hline Nausea/Vomiting & $17(59)$ & $2(7)$ \\
\hline Abdominal pain & $12(41)$ & $3(10)$ \\
\hline Constipation & $2(7)$ & - \\
\hline Diarrhea & $16(55)$ & $1(3)$ \\
\hline Melena & $1(3)$ & - \\
\hline Hypertension & $19(66)$ & $2(7)$ \\
\hline QT prolongation & $1(3)$ & $1(3)$ \\
\hline URI symptoms & $2(7)$ & - \\
\hline Pleural effusion & $2(7)$ & - \\
\hline Dyspnea & $3(10)$ & - \\
\hline Pneumonia & $1(3)$ & $1(3)$ \\
\hline Edema & $10(34)$ & - \\
\hline Skin rash & $3(10)$ & - \\
\hline Pruritus & $1(3)$ & - \\
\hline Hand-foot syndrome & $9(31)$ & - \\
\hline Alopecia & $7(24)$ & - \\
\hline Hair color change & $17(59)$ & - \\
\hline Insomnia & $2(7)$ & - \\
\hline Depression & $1(3)$ & $1(3)$ \\
\hline Myalgia & $9(31)$ & $1(3)$ \\
\hline Fatigue & $11(38)$ & $1(3)$ \\
\hline Neuropathy & $1(3)$ & - \\
\hline Headache & $4(14)$ & - \\
\hline \multicolumn{3}{|l|}{ Laboratory abnormalities } \\
\hline LFT dysfunction & $13(45)$ & $7(24)$ \\
\hline Proteinuria & $1(3)$ & $1(3)$ \\
\hline Hyperkalemia & $2(7)$ & $1(3)$ \\
\hline Hyponatremia & $2(7)$ & $1(3)$ \\
\hline Hypocalcemia & $1(3)$ & - \\
\hline Renal impairment & $2(7)$ & - \\
\hline Hypothyroidism & $3(10)$ & $1(3)$ \\
\hline Hyperlipidemia & $1(3)$ & - \\
\hline Anemia & $5(17)$ & $2(7)$ \\
\hline Leukopenia & $4(14)$ & $2(7)$ \\
\hline Thrombocytopenia & $7(24)$ & $3(10)$ \\
\hline
\end{tabular}

Values are presented as number (\%). URI, upper respiratory infection; LFT, liver function test.

ered the association between microperforation and pazopanib low. In previous trials, gastrointestinal (GI) perforation was a rare $(<1 \%) \mathrm{AE}$ associated with pazopanib and most cases were GI perforation caused by formation of a fistula in patients who showed a tumor response [25]. The patient in our study had only bone metastasis at diagnosis and the treatment response was SD. Also, in the meta-analysis, the use of VEGFR-TKIs does not seem to increase the risk of GI perforation compared with controls [26]. Thus, we suspect that this case did not represent treatment-related mortality. These safety profiles seems to be consistent with the results of previous studies of pazopanib in patients with metastatic RCC [9-11]. Therefore, pazopanib treatment was considered to be tolerable in patients with metastatic nccRCC.

This study demonstrated promising activity of pazopanib in patients with metastatic nccRCC. Although most patients experienced AEs, pazopanib was tolerated in these patients. Further investigations are needed to evaluate the clinical efficacy of pazopanib according to histologic subtype and risk group among patients with metastatic nccRCC.

\section{Electronic Supplementary Material}

Supplementary materials are available at Cancer Research and Treatment website (http://www.e-crt.org).

\section{Conflicts of Interest}

Conflict of interest relevant to this article was not reported.

\section{Author Details}

${ }^{1}$ Division of Hematology and Oncology, Department of Medicine, Samsung Medical Center, Sungkyunkwan University School of Medicine, Seoul, ${ }^{2}$ Department of Oncology, Asan Medical Center, University of Ulsan College of Medicine, Seoul, ${ }^{3}$ Division of Hematology and Oncology, Seoul National University Hospital, Seoul, ${ }^{4}$ Division of Hematology and Oncology, Gangnam Severance Hospital, Yonsei University College of Medicine, Seoul, ${ }^{5}$ Division of Hematology and Oncology, Department of Internal Medicine, Gyeongsang National University Hospital, Gyeongsang National University School of Medicine, Jinju, ${ }^{6}$ Division of Hematology and Oncology, Dong-A University Medical Center, Busan, ${ }^{7}$ Division of Medical Oncology, Department of Internal Medicine, Yonsei Cancer Center, Yonsei University College of Medicine, Seoul, ${ }^{8}$ Division of Oncology-Hematology, Department of Medicine, Yeungnam University College of Medicine, Daegu, ${ }^{9}$ Department of Internal Medicine, Hallym University Sacred Heart Hospital, Hallym University College of Medicine, Anyang, Korea 


\section{References}

1. Leibovich BC, Lohse CM, Crispen PL, Boorjian SA, Thompson $\mathrm{RH}$, Blute ML, et al. Histological subtype is an independent predictor of outcome for patients with renal cell carcinoma. J Urol. 2010;183:1309-15.

2. Motzer RJ, Bacik J, Mariani T, Russo P, Mazumdar M, Reuter $\mathrm{V}$. Treatment outcome and survival associated with metastatic renal cell carcinoma of non-clear-cell histology. J Clin Oncol. 2002;20:2376-81.

3. Vera-Badillo FE, Templeton AJ, Duran I, Ocana A, de Gouveia $\mathrm{P}$, Aneja P, et al. Systemic therapy for non-clear cell renal cell carcinomas: a systematic review and meta-analysis. Eur Urol. 2015;67:740-9.

4. National Comprehensive Cancer Network. NCCN clinical practice guidelines in oncology, kidney cancer. version 2. 2016 [Internet]. Fort Washington, PA: National Comprehensive Cancer Network; 2016 [cited 2016 Oct 20]. Available from: https://www.nccn.org/professionals/physician_gls/pdf/kid ney.pdf.

5. Dutcher JP, de Souza P, McDermott D, Figlin RA, Berkenblit A, Thiele A, et al. Effect of temsirolimus versus interferonalpha on outcome of patients with advanced renal cell carcinoma of different tumor histologies. Med Oncol. 2009;26:202-9.

6. Hudes G, Carducci M, Tomczak P, Dutcher J, Figlin R, Kapoor A, et al. Temsirolimus, interferon alfa, or both for advanced renal-cell carcinoma. N Engl J Med. 2007;356:2271-81.

7. Heng DY, Bukowski RM. Anti-angiogenic targets in the treatment of advanced renal cell carcinoma. Curr Cancer Drug Targets. 2008;8:676-82.

8. Rini BI. Vascular endothelial growth factor-targeted therapy in metastatic renal cell carcinoma. Cancer. 2009;115(10 Suppl): 2306-12.

9. Sternberg CN, Davis ID, Mardiak J, Szczylik C, Lee E, Wagstaff J, et al. Pazopanib in locally advanced or metastatic renal cell carcinoma: results of a randomized phase III trial. J Clin Oncol. 2010;28:1061-8.

10. Sternberg CN, Hawkins RE, Wagstaff J, Salman P, Mardiak J, Barrios $\mathrm{CH}$, et al. A randomised, double-blind phase III study of pazopanib in patients with advanced and/or metastatic renal cell carcinoma: final overall survival results and safety update. Eur J Cancer. 2013;49:1287-96.

11. Motzer RJ, Hutson TE, Cella D, Reeves J, Hawkins R, Guo J, et al. Pazopanib versus sunitinib in metastatic renal-cell carcinoma. N Engl J Med. 2013;369:722-31.

12. Altorki N, Lane ME, Bauer T, Lee PC, Guarino MJ, Pass H, et al. Phase II proof-of-concept study of pazopanib monotherapy in treatment-naive patients with stage I/II resectable nonsmall-cell lung cancer. J Clin Oncol. 2010;28:3131-7.

13. Friedlander M, Hancock KC, Rischin D, Messing MJ, Stringer CA, Matthys GM, et al. A Phase II, open-label study evaluating pazopanib in patients with recurrent ovarian cancer. Gynecol Oncol. 2010;119:32-7.
14. van der Graaf WT, Blay JY, Chawla SP, Kim DW, Bui-Nguyen B, Casali PG, et al. Pazopanib for metastatic soft-tissue sarcoma (PALETTE): a randomised, double-blind, placebo-controlled phase 3 trial. Lancet. 2012;379:1879-86.

15. Gore ME, Szczylik C, Porta C, Bracarda S, Bjarnason GA, Oudard $S$, et al. Final results from the large sunitinib global expanded-access trial in metastatic renal cell carcinoma. Br J Cancer. 2015;113:12-9.

16. Choueiri TK, Plantade A, Elson P, Negrier S, Ravaud A, Oudard $S$, et al. Efficacy of sunitinib and sorafenib in metastatic papillary and chromophobe renal cell carcinoma. J Clin Oncol. 2008;26:127-31.

17. Lee JL, Ahn JH, Lim HY, Park SH, Lee SH, Kim TM, et al. Multicenter phase II study of sunitinib in patients with non-clear cell renal cell carcinoma. Ann Oncol. 2012;23:2108-14.

18. Matrana MR, Baiomy A, Campbell M, Alamri S, Shetty A, Teegavarapu $\mathrm{P}$, et al. Outcomes of patients with metastatic nonclear-cell renal cell carcinoma treated with pazopanib. Clin Genitourin Cancer. 2017;15:e205-8.

19. Motzer RJ, Mazumdar M, Bacik J, Berg W, Amsterdam A, Ferrara J. Survival and prognostic stratification of 670 patients with advanced renal cell carcinoma. J Clin Oncol. 1999;17: 2530-40.

20. Koh Y, Lim HY, Ahn JH, Lee JL, Rha SY, Kim YJ, et al. Phase II trial of everolimus for the treatment of nonclear-cell renal cell carcinoma. Ann Oncol. 2013;24:1026-31.

21. Gordon MS, Hussey M, Nagle RB, Lara PN Jr, Mack PC, Dutcher J, et al. Phase II study of erlotinib in patients with locally advanced or metastatic papillary histology renal cell cancer: SWOG S0317. J Clin Oncol. 2009;27:5788-93.

22. Armstrong AJ, Halabi S, Eisen T, Broderick S, Stadler WM, Jones RJ, et al. Everolimus versus sunitinib for patients with metastatic non-clear cell renal cell carcinoma (ASPEN): a multicentre, open-label, randomised phase 2 trial. Lancet Oncol. 2016;17:378-88.

23. Sankin A, Hakimi AA, Hsieh JJ, Molina AM. Metastatic nonclear cell renal cell carcinoma: an evidence based review of current treatment strategies. Front Oncol. 2015;5:67.

24. Tannir NM, Plimack E, Ng C, Tamboli P, Bekele NB, Xiao L, et al. A phase 2 trial of sunitinib in patients with advanced non-clear cell renal cell carcinoma. Eur Urol. 2012;62:1013-9.

25. Necchi A, Mariani L, Zaffaroni N, Schwartz LH, Giannatempo $\mathrm{P}$, Crippa F, et al. Pazopanib in advanced and platinum-resistant urothelial cancer: an open-label, single group, phase 2 trial. Lancet Oncol. 2012;13:810-6.

26. Qi WX, Sun YJ, Tang LN, Shen Z, Yao Y. Risk of gastrointestinal perforation in cancer patients treated with vascular endothelial growth factor receptor tyrosine kinase inhibitors: a systematic review and meta-analysis. Crit Rev Oncol Hematol. 2014;89:394-403. 